
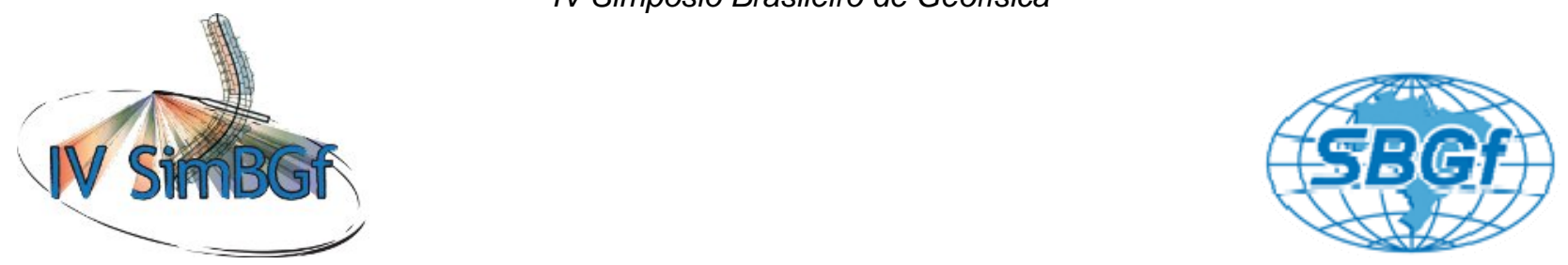

\title{
Migrações pós e pré-empilhamento de dados sísmicos registrados sobre o talude continental da Bacia do Jequitinhonha
}

Pedro H. S. Sales, Tiago S. Cabral e Marco A. B. Botelho, Universidade Federal da Bahia

Copyright 2010, SBGf - Sociedade Brasileira de Geofísica

Este texto foi preparado para a apresentação no IV Simpósio Brasileiro de Geofísica, Brasília, 14 a 17 de novembro de 2010. Seu conteúdo foi revisado pelo Comitế Técnico do IV SimBGf, mas não necessariamente representa a opinião da SBGf ou de seus associados. É proibida a reprodução total ou parcial deste material para propósitos comerciais sem prévia autorização da SBG

\section{Resumo}

Três métodos de migração em profundidade foram aplicados em seções empilhadas registradas sobre o talude continental da Bacia do Jequitinhonha. Os métodos empregados na migração foram a migração reversa no tempo (Reverse Time Migration ou RTM) empregando operadores de diferenças finitas, a migração Phase-Shift Plus Interpolation (PSPI) e a migração splitstep. Tais migrações pertencem ao Pacote SU (Seismic Unix) desenvolvido pelo Laboratório CWP (Center for Wave Phenomena) da Colorado School of Mines. Desenvolvemos um programa em linguagem Fortran que implementa a migração RTM utilizando operadores de diferenças finitas. Vale ressaltar que a RTM foi aplicada tanto em seções empilhadas como em famílias de tiro comum. Os campos de velocidades utilizados nas migrações foram obtidos a partir da análise de velocidades clássica. Os resultados obtidos com os diferentes métodos de migração são comparados entre si, verificando-se a melhor resolução e continuidade dos refletores provenientes dos métodos espectrais, permitindo uma análise interpretativa mais apurada.

\section{Introdução}

O método geofísico que fornece a maior resolução na definição de armadilhas de hidrocarbonetos é o método sísmico de reflexão. Entretanto, somente com o avanço dos recursos computacionais foi possível aperfeiçoar técnicas que permitam uma definição mais precisa das estruturas em subsuperfície. $\mathrm{Na}$ sequência de processamento de dados sísmicos de reflexão existe uma etapa denominada migração, a qual é responsável por remover efeitos associados a propagação do campo de ondas.

O produto final do processamento CMP é a seção empilhada, a qual ainda não pode ser considerada como uma imagem perfeita da subsuperfície. Esta seção empilhada deverá ser migrada para que qualquer estrutura curva ou inclinada ocupe a verdadeira posição espacial e apresente a sua verdadeira dimensão. Este trabalho inicia com o processamento CMP clássico dos dados sísmicos de reflexão, utilizando os dados de levantamentos sísmicos marinhos realizados na Bacia do
Jequitinhonha (Litoral do Estado da Bahia), os quais serão processados usando o Pacote SU. Na etapa seguinte, foram aplicadas migrações que empregam operadores de diferenças finitas (no tempo e também no espaço), cujos resultados são comparados com os obtidos das migrações split-step e PSPI.

A RMT também é executada por meio de um programa Fortran que utiliza operadores de diferenças finitas na resolução das derivadas parciais da equação da onda. Foram utilizados operadores de segunda ordem para solucionar as derivadas temporais e de quarta ordem para as derivadas espaciais. A migração RTM também foi aplicada em famílias de tiro comum e os resultados comparados com o obtido a partir da seção empilhada.

\section{A Bacia do Jequitinhonha}

A Bacia do Jequitinhonha está localizada na porção nordeste da margem leste brasileira, mais precisamente no litoral sul da Bahia, em frente à foz do rio Jequitinhonha.

Essa bacia se encontra na borda sul do Cráton do São Francisco, entre os paralelos $-14^{\circ} 35^{\prime}$ e $-16^{\circ} 29^{\prime}$ e os meridianos $-39^{\circ} 33^{\prime}$ e $-37^{\circ} 21^{\prime}$, sendo constituída por terrenos dominantemente granulíticos, total ou parcialmente retrabalhados no ciclo transamazônico, segundo. Ela é limitada ao norte pela Bacia de CamamuAlmada e ao sul pela Bacia de Cumuruxatiba.

Trata-se de uma bacia sedimentar de rifte, de idade aptiana, que evoluiu para uma bacia de margem passiva. É caracterizada por uma fase inicial pré-rifte, onde predomina um estilo de sinéclise intracontinental, e por três megassequências: a megassequência rifte, constituída por sedimentos siliciclásticos e folhelhos, a megassequência transicional, constituída por evaporitos neoaptianos, e megassequência pós-rifte, constituída por sedimentos siliciclásticos depositados em leques deltaicos e por depósitos carbonáticos de alta e baixa energia nas regiões distais da bacia.

\section{Dados sísmicos}

Utilizam-se quatro linhas sísmicas de reflexão 2D, cuja localização geográfica é mostrada na Figura 1, sobre a zona de talude da Bacia do Jequitinhonha. Essas linhas pertencem à Petrobras e foram obtidas através de projetos realizados pelo Laboratório de Geofísica de Exploração de Petróleo (LAGEP) do Centro de Pesquisa 
em Geofísica e Geologia (CPGG) da Universidade Federal da Bahia.

Esses dados foram adquiridos pela equipe sísmica 214 da Petrobras no setor offshore da bacia. Desses dados, dois estão orientados na direção norte-sul (linhas 2140266 e 214-0268) e dois estão orientados na direção leste oeste (linhas 214-0297 e 214-0303).

Os arquivos de dados foram disponibilizados no formato SEG-Y. Em todas as linhas, foram usados 120 canais, tendo uma separação entre eles de $25 \mathrm{~m}$ e um afastamento mínimo de $150 \mathrm{~m}$. O arranjo utilizado foi o end-on. Os dados foram adquiridos a uma taxa de amostragem de 4 milissegundos, e o número total de amostras por traço é 1751.

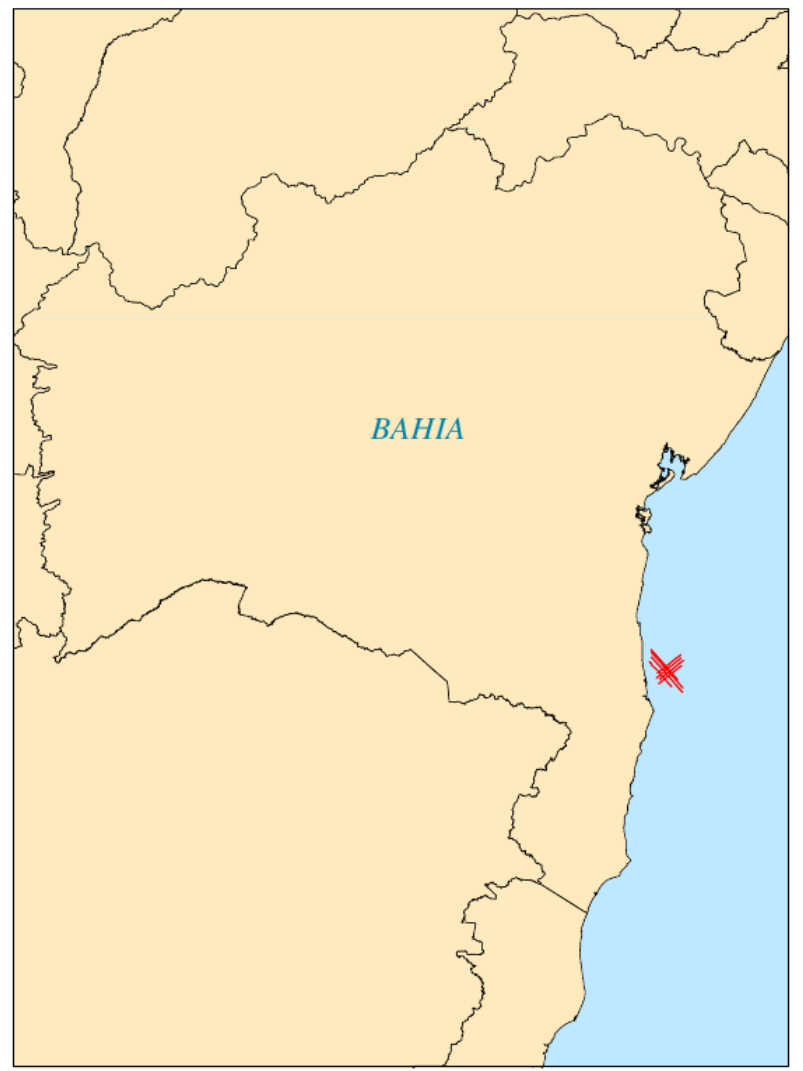

Figura 1 - Localização geográfica dos dados utilizados no trabalho.

\section{Processamento sísmico}

O processamento dos dados sísmicos foi feito utilizandose o pacote livre Seismic Unix (SU) do Laboratório Center of Wave Phenomena (CWP) da Colorado School of Mines (Cohen, 2002). Para obter as seções empilhadas, foram aplicadas principais as etapas do processamento CMP clássico.

A primeira etapa foi o pré-processamento, que incluiu a importação dos dados (conversão do formato padrão SEG-Y para o formato do pacote SU), a definição de geometria e a edição.
Observou-se que os dados apresentam conteúdos de frequência semelhantes, por isso foi aplicado um mesmo filtro passa-banda a todos eles. Os valores de frequência utilizados no filtro trapezoidal foram 15, 20, 45 e $50 \mathrm{~Hz}$.

Para a definição do campo de velocidades, foram realizadas análises a cada 100 CMPs. A Figura 2 ilustra a análise de velocidades do CMP 370 da seção 214-0297. É válido observar que existem eventos de alta amplitude que fornecem informações a baixa velocidades (por exemplo Pode-se observar como a presença de eventos de alta amplitude correspondentes a múltiplas (que geram picos de velocidade mais baixa no espectro) dificultam a análise.

O campo de velocidades RMS obtido a partir das análises dos CMPs foi, posteriormente, suavizado, pois se sabe que em zona íngremes a interpolação de CMPs muito afastados gera maiores imprecisões. Em seguida, ele foi convertido em um campo de velocidades intervalares, que é necessário para realizar as migrações. A Figura 3 mostra que o campo de velocidades final obtido para a linha sísmica 214-0297, o qual apresenta velocidades crescentes com a profundidade.

Na Figura 4, observa-se a citada seção empilhada, obtida a partir do campo de velocidades mostrado na figura 3 . Os refletores, em sua maioria, estão bem definidos, indicando que o campo de velocidades permitiu um empilhamento construtivo dos refletores.

A fim de colapsar as hipérboles de difração e para posicionar corretamente os refletores serão feitas migrações pré e pós-empilhamento utilizando programas do pacote SU. Por fim, aplicou-se o programa desenvolvido em Fortran de migração pós-empilhamento por diferenças finitas (Figura 8), para que seus resultados possam ser comparados com os obtidos a partir do pacote SU.

\section{Migração de dados sísmicos}

O conceito de refletor explosivo, vide Loewenthal et al. (1976), permite que se empregue a equação da onda para extrapolar de forma reversa no tempo o campo de ondas registrados na superfície $(x, z=0, t)$, o que seria a nossa seção empilhada, oriunda do processamento CMP, até os pontos da subsuperfície onde cada sinal sísmico foi gerado (fonte explosiva), ou melhor, onde ocorreu a reflexão. Dessa forma, é gerada a nossa seção migrada $(x, z, t=0)$.

A migração de seções empilhadas pode ser feita por diferentes métodos que, na verdade, só diferem na técnica numérica utilizada para resolver as derivadas parciais presentes na equação acústica da onda.

O método de migração que utiliza o conceito do refletor explosivo e realiza a operação acima descrita é conhecido como migração reversa no tempo, RTM (Baysal et al., 1983), que extrapola um plano $x-z$ para trás no tempo, tomando o campo de pressão $P(x, z=0, t)$ como uma condição de contorno. Assim, em cada passo de tempo, calculam-se os instantâneos do plano $x-z$ em 
tempos diferentes. No tempo $t=0$, este plano $x-z$ será o resultado da migração de $P(x, z, t=0)$. No presente trabalho, foi utilizado um algoritmo que emprega diferenças finitas para resolver a equação da onda e realizar a retropropagação temporal. Vide ainda os trabalhos de Botelho (1986) e Loewenthal et al. (1985).

A migração RTM de dados sísmicos não empilhados foi apresentada por Faria (1986) e, nestes casos, o tempo de imagem corresponde ao princípio de coincidência dos tempos estabelecido por Claerbout (1971). Neste trabalho também realizamos migrações das famílias de tiro comum.

Outra forma de resolver a equação da onda é apresentada em (Gazdag \& Sguazzero, 1984), que usa o método de rotação de fase mais interpolação (Phase Shift Plus Interpolation ou PSPI), onde o passo é tomado na dimensão de profundidade. Através da propriedade da transformada de Fourier que relaciona um deslocamento no domínio espacial com uma mudança de fase no domínio Fourier, os valores em uma profundidade podem ser extraídos a partir dos valores em outra através de uma operação que envolve o conhecimento da distância entre elas. A menos da necessidade de interpolação, caso exista mais de uma velocidade de referência, o algoritmo transcorre totalmente no domínio da transformada Fourier. O passo em profundidade deve ser feito para cada velocidade existente entre os dois níveis (ou ao menos um pequeno grupo delas capaz de representá-las), pois a solução só é correta para o caso de velocidade constante. Vide os trabalhos de Gazdag (1978) e Gazdag e Squazzero (1984).

O método split-step foi apresentado pelos trabalhos de Freire (1988) e Stoffa et al. (1990), onde procura burlar a dificuldade enfrentada pelo método PSPI quando diante de múltiplas velocidades de referência. A formulação separa o campo de vagarosidades (definindo vagarosidade $\mathrm{s}$ como $\mathrm{o}$ inverso de velocidade $\mathrm{v}$ ) horizontalmente variantes em um termo médio e um termo de perturbação. A equação da onda é solucionada para a vagarosidade média e posteriormente corrigida pelo termo de perturbação. Entretanto, o método impõe como condição uma amplitude satisfatoriamente pequena para o termo de perturbação, para que possa ser descartado seu termo de segunda ordem na solução.

\section{Resultados}

O trecho correspondente ao Talude da Bacia do Jequitinhonha linha 214-0297 apresenta dois fortes refletores a aproximadamente 1,8 e $2,0 \mathrm{~s}$ e uma estrutura diapírica próxima ao CMP 80.

A seção migrada com a técnica PSPI aparenta possuir um maior conteúdo de freqüência e fornece uma melhor continuidade dos refletores. As migrações usando operadores de diferenças finitas, particularmente 0 implementado em Fortran, tem menor conteúdo de freqüência $e$, este último necessita de modificações (diminuição) no Dz e Dt pois apresenta fortes índices de dispersão numérica.
A estrutura com aspecto de um diápiro no início da seção tem as difrações colapsadas e seu topo convexo reduzido em dimensão, como era de se esperar, em todas as três migrações.

A linha 214-0266 apresenta uma estrutura côncava, muito provavelmente associada a um diápiro de sal, porém a linha está truncada e não temos a imagem do sal.Aplicamos a migração RTM pós e pré empilhamento nos dados sísmicos desta última linha usando os programas do pacote $\mathrm{SU}$ e podemos verificar que a estrutura côncava é corrigida em ambas as migrações.Um forte refletor inclinado que mergulha de $2.000 \mathrm{~m}$ no início da seção até $4.000 \mathrm{~m}$ no fim da seção é bem imageado em ambas as seções migradas.

\section{Agradecimentos}

Os dois primeiros autores agradecem ao CNPq pelas bolsas de iniciação científica recebidas, as quais foram alocadas à Rede CTPETRO de Geofísica de Exploração correspondente ao convênio Imageamento Sísmico do Talude Continental UFBA-FINEP-Petrobras-FAPEX.

\section{Referências}

BAYSAL E, KOSLOFF DD \& SHERWOOD J. 1983. Reverse time migration. Geophysics, Society of Exploration Geophysicists, 48:1514-1524.

BOTELHO MAB. 1986. Modelamento sísmico na Bacia do Recôncavo usando a técnica de traçamento dos raios Universidade Federal da Bahia, Tese de Doutorado.

COHEN JE \& Jr. JS. 2002. The New SU User's Manual, Colorado School of Mines.

FARIA, EL. 1986. Migração antes do empilhamento utilizando propagação reversa no tempo. Universidade Federal da Bahia, Dissertação de Mestrado.

FREIRE RML. 1988. Migração por mudança de fase em duas etapas. Universidade Federal da Bahia, Tese de Doutorado.

GAZDAG J. 1978. Wave equation migration with the phase-shift method: Geophysics, Society of Exploration Geophysicists, 43:1342-1351.

GAZDAG J \& SQUAZZERO P. 1984. Migration of seismic data by phase shift plus interpolation. Geophysics, Society of Exploration Geophysicists, 49:124-131.

LOEWENTHAL D, LU L, ROBERSON R \& SHERWOOD JWC. 1976. The wave equation applied to migration. Geophys. Prospecting, 24:380-399.

LOEWENTHAL D, WANG CJ \& JOHNSON OG. 1985. High order finite difference modeling and reverse time migration. Exploration Geophysics, 22:533-545.

STOFFA PL, FOKKEMA JT, FREIRE RML \& KESSINGER WP. 1990. Split-Step Fourier migration. Geophysics, Society of Exploration Geophysicists. 55: 410-421. 


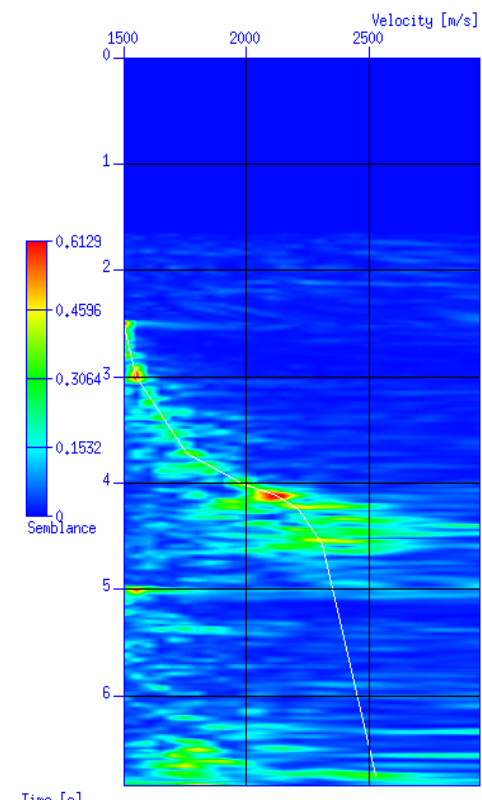

Semblance Plot CMP 370

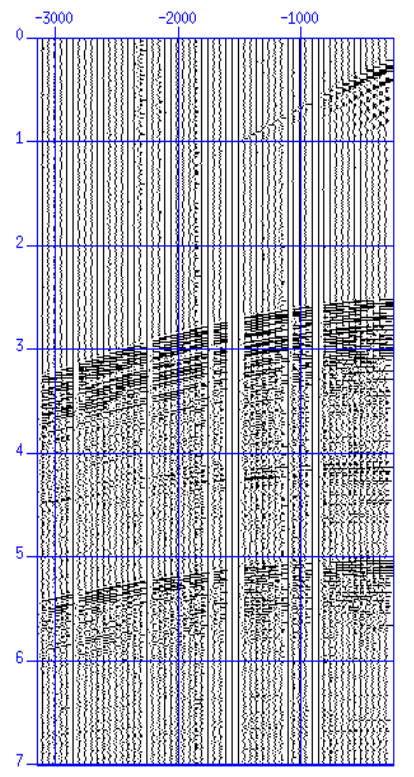

CMP gather 370

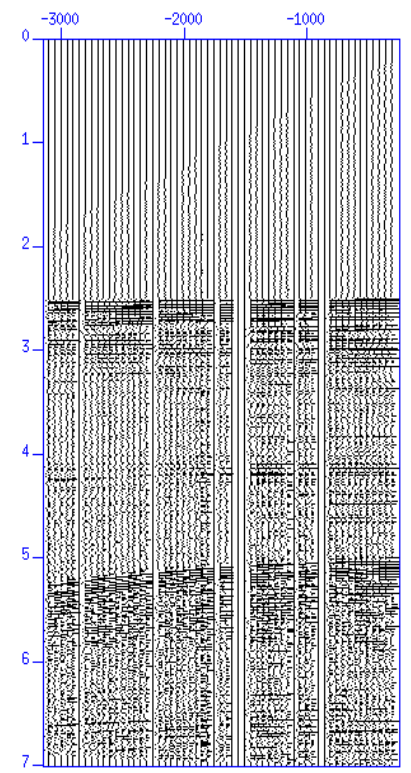

CMP after NHO correction

Figura 2 - Interface utilizada para a análise de velocidades. Pode-se ver o espectro de velocidade (à esquerda), a família CMP antes da correção NMO (ao centro) e após a correção de NMO (à direita).

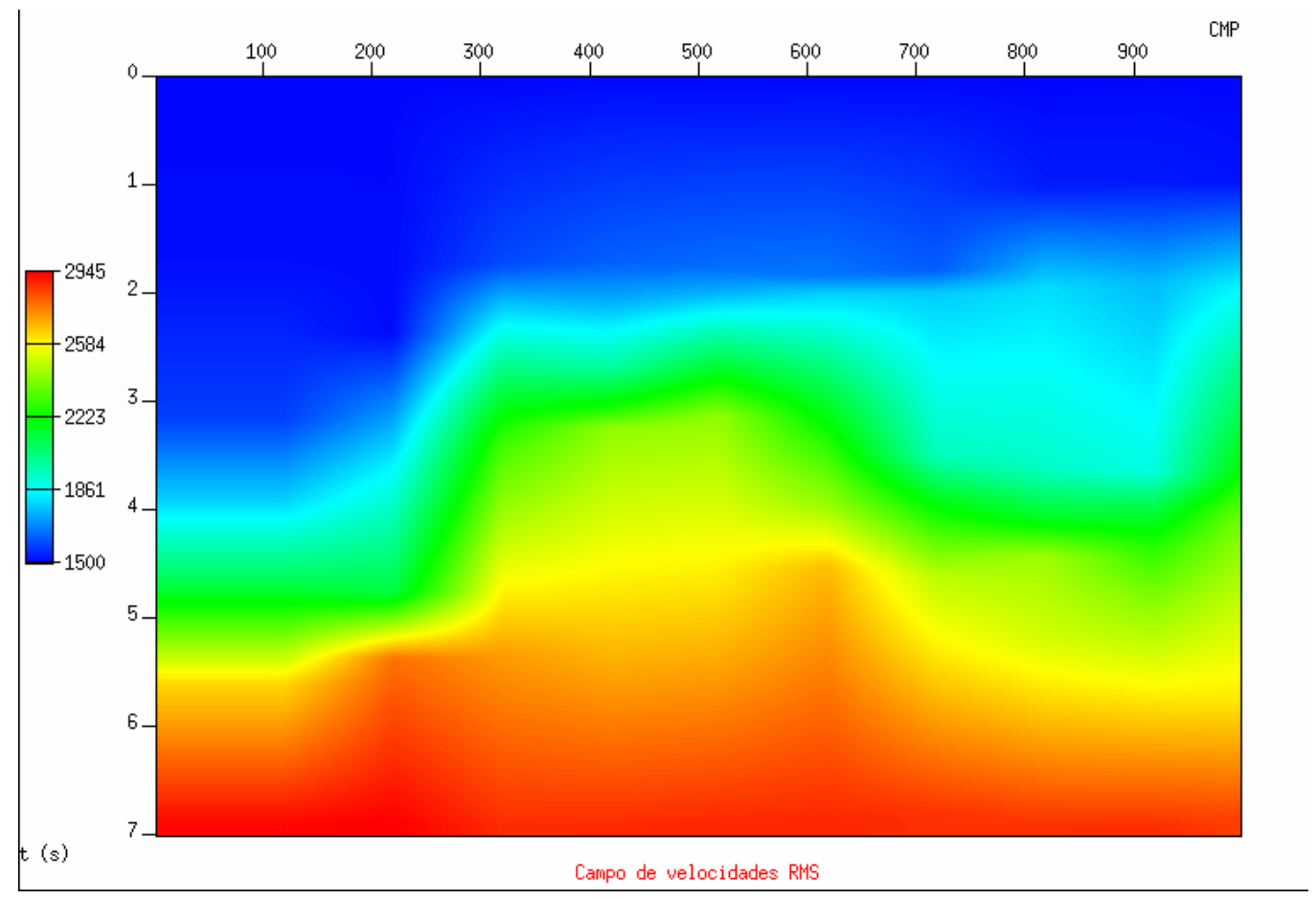

Figura 3 -Campo de velocidades utilizado para realizar o empilhamento e as migrações do dado 214-0297. 


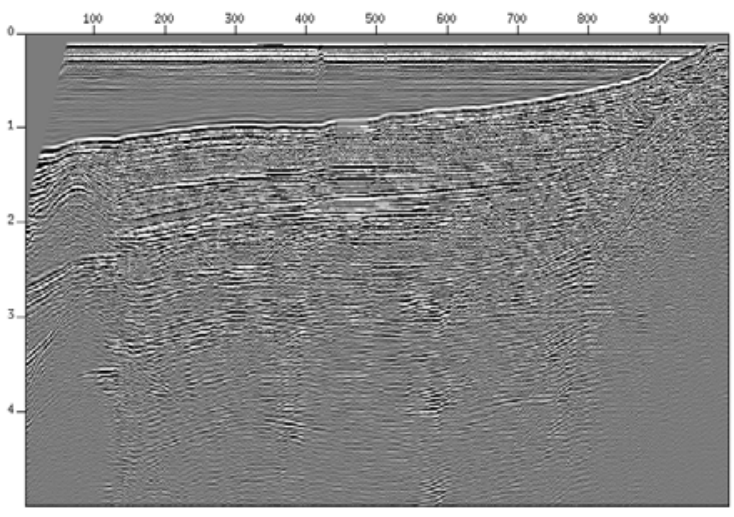

Figura 4 -Seção empilhada correspondente a linha 214-0297,após a aplicação de um filtro de freqüência trapezoidal cujas freqüências são de 15,20,45 e $50 \mathrm{~Hz} A$ seção possui 1000 traços, sendo o intervalo entre traços de $25 \mathrm{~m}$. Cada traço possui 1751 amostras, sendo o intervalo de amostragem de $4 \mathrm{~ms}$.

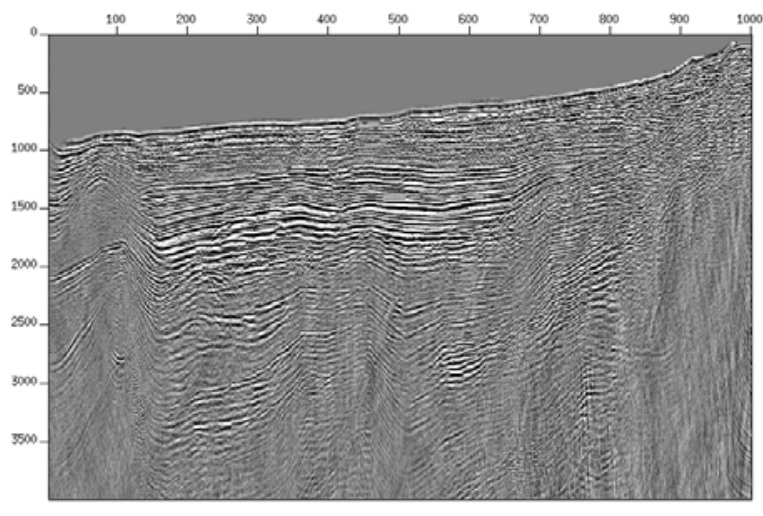

Figura 5 -Seção migrada por diferenças finitas correspondente a linha 214-0297 usando $D x=25 m$ e $D z=7 m$. $O$ programa utilizado pertence ao pacote SU.

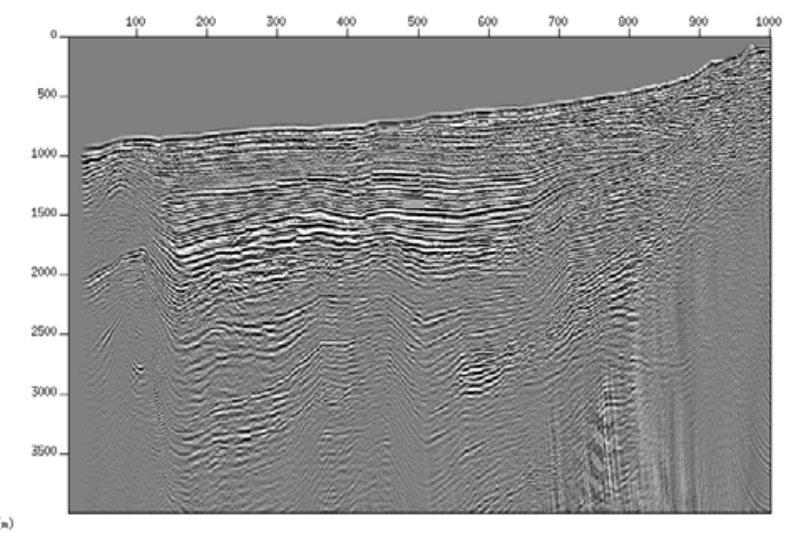

Figura 6-Seção migrada com a técnica PSPI,correspondente a linha 214-0297 usando Dx=25m e Dz=7m. O programa utilizado pertence ao pacote SU. 


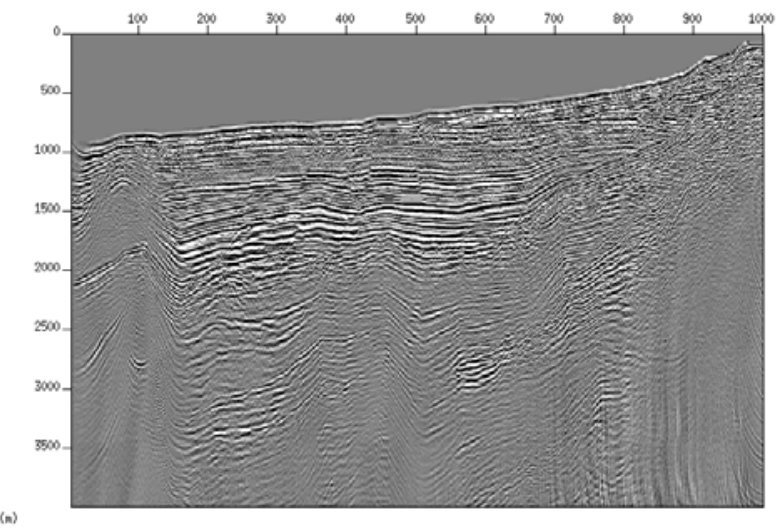

Figura 7 - Seção migrada com a técnica split-step,correspondente a linha 214-0297 usando $D x=25 \mathrm{~m}$ e $D z=7 \mathrm{~m} .0$ programa utilizado pertence ao pacote SU..

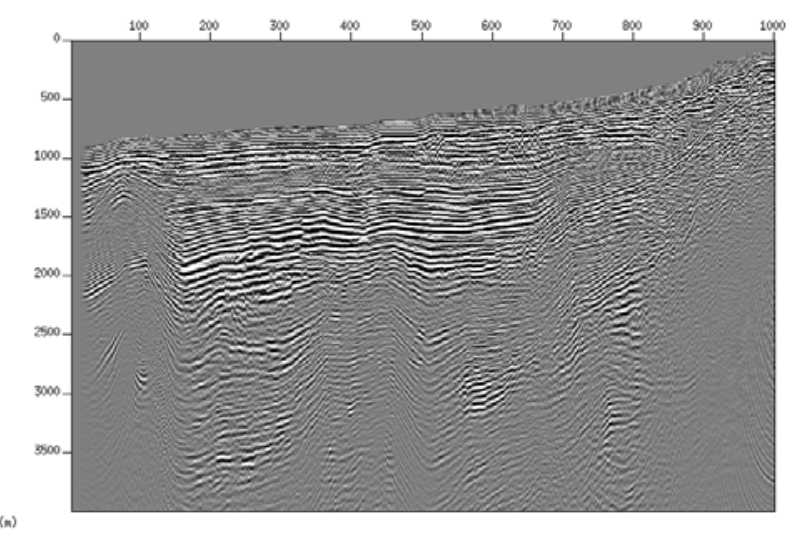

Figura 8 -Seção após a migração reversa no tempo por diferenças finitas correspondente a linha 214-0297 implementada através do programa Fortran.

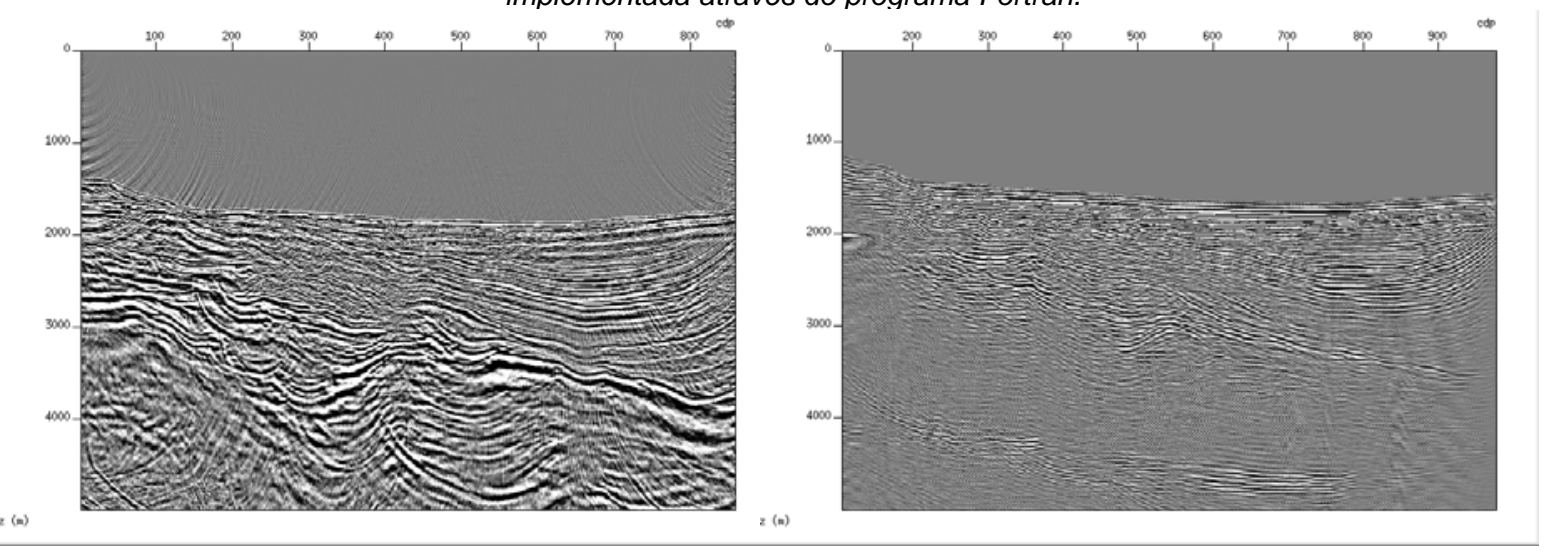

Figura 9 -Linha sísmica 214-0266: (a) Seção empilhada e migrada usando a RTM do pacote SU (Dx=25m,Dz=7m e $D t=4 \mathrm{~ms}$ ); (b)Seção migrada antes do empilhamento usando a $R T M$ pré-stack do pacote $S U$ ( $D x=25 \mathrm{~m}, D z=7 \mathrm{~m} e$ $D t=2 m s)$. 